\title{
LETTERS
}

\section{The College of Physicians and Surgeons of Ontario on MAiD referrals}

The College of Physicians and Surgeons of Ontario (CPSO) was at the forefront of policy development as the legislation on medical assistance in dying (MAiD) was coming into force. We provided muchneeded guidance to the profession on this important issue after consulting broadly with the public and the profession.

In a recent editorial, ${ }^{1}$ Dr. Kelsall mischaracterized the MAiD discussion as it pertains to the "effective referral" provision of our MAiD policy for doctors who object to MAiD, and misunderstood what constitutes "complicity" when objecting doctors encounter patients seeking help to end their lives.

An effective referral is not synonymous with a direct referral, and there are a number of ways that objecting physicians could discharge their obligations of effective referral without relying on a direct referral, as we have made clear in our MAiD policy's accompanying fact sheet. ${ }^{2}$ For example, objecting physicians can make the referral themselves or delegate the task to a staff member or colleague; the physician or a designate can connect the patient with an agency charged with facilitating referrals for the health care service (such as Ontario's care coordination service for MAiD) and make arrangements for the agency to see that patient; and doctors who work as part of a practice team can establish triage systems that ensure the objecting physician would never have to see a patient seeking MAiD.
Of note is that an effective referral, in any clinical situation, does not guarantee a specific outcome for a patient. To receive MAiD, the patient must be found eligible in accordance with criteria in the Criminal Code by two independent clinicians, the patient must consent to MAiD and all of the safeguards set out in the Criminal Code must be met. The chief goal of our effective referral provision is to help patients find a pathway to pursue their interest in MAiD and to get help in that regard.

It is unfortunate that Dr. Kelsall chose to frame the broader discussion as a scenario of doctors versus patients. The goal of the college's policy is to respect patients and physicians. It seeks to ensure all patients - including the most vulnerable - have equitable access to available health care services in Ontario, while respecting physicians' beliefs and religious freedoms.

We are not concerned simply with "the basics of physician professionalism." ${ }^{1}$ Our policy is rooted in the core values of the medical profession, such as service, altruism, trustworthiness and compassion, articulated in our Practice Guide. ${ }^{3}$ Our effective referral provision is in place to ensure that objecting physicians do not abandon their patients and that even the most vulnerable members of society have equitable access to our public health care system. As Dr. Danielle Martin said in an affidavit quoted in the Divisional Court decision, "The need for family physicians to provide patients with information and meaningful referrals is espe- cially important in the Canadian system because many citizens do not have other access points through which to enter the system, or are unaware of what other access points exist. ... [T] he geographic and community-based nature of our primary care catchment makes access to care particularly dependent on the consistency of service provided by doctors." 4

Doctors have a fiduciary responsibility to all of their patients no matter where they are in their life cycle, including those who are grievously and irremediably ill and wish to seek assistance in ending their lives. The CPSO was gratified that the court recognized the importance of effective referral for patients.

\section{Steven C. Bodley MD}

President, The College of Physicians and Surgeons of Ontario, Toronto, Ont.

Cite as: CMAJ 2018 June 11;190:E724. doi: 10.1503/cmaj.69469

\section{References}

1. Kelsall D. Physicians are not solely responsible for ensuring access to medical assistance in dying. CMAJ 2018;190:E181.

2. Fact sheet - ensuring access to care: effective referral. Toronto: The College of Physicians and Surgeons of Ontario; 2016. Available: www.cpso. on.ca/CPSO/media/documents/Policies/Policy -Items/PAD-Effective-Referral-FactSheet.pdf (accessed 2018 Feb. 21).

3. The practice guide: medical professionalism and college policies. Toronto: The College of Physicians and Surgeons of Ontario; 2007.

4. The Christian Medical and Dental Society of Canada v. College of Physicians and Surgeons of Ontario, 2018 ONSC 579.

Competing interests: None declared. 